\title{
Os rankings acadêmicos e suas relações com os ODS: estudo de caso na Universidade Federal do Tocantins
}

\author{
Michele Silva Costa Sousa ${ }^{1}$ \\ Waldecy Rodrigues ${ }^{2}$ \\ Airton Cançado ${ }^{3}$
}

Submissão: 18/09/2021

Aceite: $10 / 12 / 2021$

\begin{abstract}
Resumo
Desde os anos 2000, proliferou a criação de rankings acadêmicos, cada qual com sua metodologia, uso de critérios e métricas na tentativa de direcionar o que se considera qualidade no ensino superior. No entanto, nem sempre é possível mensurar de forma equitativa as universidades. Uma alternativa criada foi o Times Higher Education Impact Rankings com a proposta de avaliar o impacto das universidades no combate à pobreza, o fomento a igualdade de gênero e ações de mitigação climática, dentre outras temáticas atreladas as metas dos Objetivos de Desenvolvimento Sustentável (ODS). Neste contexto, o objetivo deste estudo é analisar os rankings acadêmicos e suas relações com os ODS em um estudo de caso na Universidade Federal do Tocantins (UFT), localizada na região Norte brasileira, por meio da análise de documentos secundários. A conclusão que se chega é que mesmo diante da criação de um ranking atrelado aos ODS, a UFT e as universidades da região Norte continuam a serem excluídas. $O$ resultado apresenta que estas instituições não conseguem ainda se enquadrar nos padrões propostos pelo ranking.
\end{abstract}

Palavras-chave: Indicadores. Ensino Superior. Internacionalização. Pós-graduação. Desenvolvimento Regional.

\section{Academic rankings and their relations with the SDGs: a case study at the Federal University of Tocantins}

\begin{abstract}
Since the 2000s, the creation of academic rankings has proliferated, each with its own methodology, use of criteria and metrics in an attempt to direct what it considers quality in higher education. However, it is not always possible to measure universities equitably. An alternative created was the Times Higher Education Impact Rankings with the proposal to assess the impact of universities on fighting poverty, promoting gender equality and climate mitigation actions, among other topics linked to the goals of the Sustainable Development Goals (SDGs). In this context, the objective of this study is to analyze the academic rankings and their relationship with the SDGs in a case study at the Federal University of Tocantins (UFT), located in the northern region of Brazil, through the analysis of secondary documents. The conclusion reached is that even in the face of the creation of a ranking linked to the SDGs, UFT and universities in the North region continue to be excluded. The explanation is that they are still unable to meet the standards proposed by the ranking.
\end{abstract}

Keywords: Indicators. University education. Internationalization. Postgraduate studies. Regional development.

\footnotetext{
${ }^{1}$ Doutoranda em Desenvolvimento Regional no Programa de Pós-Graduação em Desenvolvimento Regional da Universidade Federal do Tocantins (UFT). http://orcid.org/0000-0002-7250-3200

Email:michele2sc@yahoo.com.br

${ }^{2}$ Doutorado em Sociologia (UnB) e Pós-Doutorado em Economia (UnB). Professor do Programa de Pós-Graduação em Desenvolvimento Regional da Universidade Federal do Tocantins (UFT). Coordenador Adjunto de Programas Profissionais da Área de Planejamento Urbano e Regional da CAPES. Email:waldecy@uft.edu.br

${ }^{3}$ Doutor em Administração (UFLA). Professor e Coordenador do Programa de Pós-Graduação em Desenvolvimento Regional da Universidade Federal do Tocantins (UFT). Email: airtoncardoso@yahoo.com.br
} 


\section{Introdução}

Os dados apresentados pelo Censo do Ensino Superior de 2019 apontam para a consolidação e expansão do ensino superior no Brasil. Das 2.608 instituições de ensino cadastradas, 79,6\% (2.076) são faculdades. No entanto, apesar deste quantitativo, nelas estão matriculadas apenas $19 \%$ do total de discentes. Por outro lado, as universidades $(7,6 \%)$ representam 52,2\% dos graduandos matriculados (INEP, 2019). Neste caso, verifica-se que a maior parte dos brasileiros matriculados no ensino superior está em uma instituição que lida com o tripé: ensino (formação humana), pesquisa (produção de conhecimento) e extensão (transferência do conhecimento gerado para a sociedade).

Outros dados apontam que no período de 2009 a 2019, o número de matrículas na educação superior aumentou em média $43,7 \%$, sendo $47,3 \%$ na rede privada e $36,5 \%$ na rede pública. Nos cursos de graduação à distância, as matrículas expandiram 192,4\%, enquanto na modalidade presencial o crescimento foi de $20,3 \%$ nesse mesmo período. Importante destacar que os estados da Paraíba, Tocantins e Rio Grande do Norte tiveram o quantitativo de matrículas na rede pública praticamente igual à rede privada. Em relação aos discentes estrangeiros matriculados no país, em 2019, constatou-se que 45,2\% são provenientes da América Latina. Já Angola foi o país que mais enviou estudantes (1.689) para cursar graduação no Brasil (INEP, 2019).

Essas estatísticas sobre o crescimento do ensino superior brasileiro foram impulsionadas graças à regulamentação em 1996 da Lei de Diretrizes e Bases da Educação (LDB) e a criação em 2004 do Sistema Nacional de Avaliação da Educação Superior (Sinaes) (BRASIL, 1996; 2004). Além disso, contribuiu o fato de existir uma relação direta entre o aumento do índice de empregabilidade e a formação acadêmica, uma vez que pessoas que não possuem diplomação são as primeiras a terem seus empregos extintos em um cenário de crise econômica (OECD, 2012). Assim, a conclusão de um curso superior tem uma representação social pautada na ascensão econômica e no acesso a uma diversidade de valores positivos propagados pela sociedade como sucesso, dinheiro e felicidade (BOULTON; LUCAS, 2011).

Diante da busca por uma trajetória de vida universitária e, por consequência, melhores perspectivas de emprego e qualidade de vida é que emergiram os rankings acadêmicos (KING, 2009). O primeiro ranking surgiu nos Estados Unidos, no ano de 2003, liderado pelo jornal 
United States News como forma de avaliar metodologias aplicadas no ensino superior e acirrar a competição entre universidades americanas por alunos estrangeiros (RIGHETTI, 2016).

Desde, então, os rankings têm se tornado, cada vez mais, recorrentes no ambiente acadêmico, o que parece inevitável na atual conjuntura. Isso por serem utilizados com diferentes propósitos, por discentes, docentes, gestores universitários, formuladores de políticas públicas, dentre outros atores envolvidos com o ensino superior a fim de analisar as classificações de suas universidades e, assim, ajustar possíveis desdobramentos em seus planejamentos e para tomadas de decisões estratégicas.

Por ora, os rankings acadêmicos estão influenciando as instituições de ensino superior a adotarem comportamento empresarial (mesmo as públicas que não visam lucro), diante da busca pela eficiência, produtividade e competitividade. A explicação deste fenômeno é que os rankings estão sendo considerados como uma espécie de avaliação de reputação e prestígio das universidades, embora não reflitam diretamente a qualidade das instituições avaliadas (LEAL; STALLIVIERI; MORAES, 2018). Seja como for, “[...] uma boa posição em um ranking é um bom argumento para uma promoção, um cartão de visitas da universidade, uma condição sine qua non para uma importante colaboração internacional" (VINCKE, 2009, p.12).

Mesmo com distintas finalidades e alvo de controvérsias por parte da sociedade, os rankings têm sido considerados parâmetros para o financiamento público e privado (PÉREZESPARRELLS; LOPEZ, 2009), indutores à competição entre as universidades (NUNES; FERNANDES, 2014), além de ser referência em termos de impactos de internacionalização, governança, autonomia, qualidade e produtividade (GONÇALVES; CALDERÓN, 2017). Isso reforça a ideia do viés produtivista acadêmico e de instrumentalismo econômico que têm caracterizado a educação superior nacional e internacional contemporânea (VIEIRA; LIMA, 2016)

Entretanto, nem sempre os indicadores de métricas selecionados nos rankings acadêmicos são claros na forma de mensuração, devido principalmente à complexidade inerente ao conceito e aos obstáculos em compreendê-los em profundidade. Isto resulta numa falta de entendimento pleno do seu significado e na carência de modelos padronizados para que as universidades ampliem sua finalidade e atinjam o patamar almejado (KNIGHT, 2015; LEAL; STALLIVIERI; MORAES, 2018).

O uso dos rankings acadêmicos como único parâmetro para a tomada de decisão de diferentes atores de determinada área e na formulação de políticas públicas de ensino superior 
é considerado equivocado, pois existem limitações significativas em termos filosóficos e pragmáticos para cada metodologia adotada (LEAL, STALLIVIERI, MORAES; 2018).

$\mathrm{Na}$ maioria dos casos, os rankings seguem sua mensuração numa perspectiva preponderantemente quantitativa. Nem sempre os indicadores e seus respectivos pesos são orientados por alguma justificativa técnica. Por isso, que, geralmente, implicam em superficialidade, visto que continuam a favorecer universidades que já são privilegiadas e consolidadas (ALTBACH, 2015). Outro viés é que na ausência de uma avaliação de caráter qualitativo e contextualizado, as universidades menores e localizadas em regiões com aguçadas desigualdades sociais e tecnológicas dificilmente alcançarão indicadores satisfatórios. ${ }^{1,2} \mathrm{Ou}$ seja, se ao formular políticas de investimentos à educação superior prevalecer a valorização de universidades de "elite" propagadas pelos rankings internacionais, isto proporcionará maiores desigualdades e concentração de recursos e esforços para a maioria das universidades brasileiras. Trata-se, assim, da penalização do sistema universitário como um todo (SANTOS; NORONHA, 2016).

É o caso das universidades presentes na região Norte do país que precisam superar as assimetrias regionais. Com $8,81 \%$ do total da população brasileira, a região Norte possui apenas $5,3 \%$ do total de doutores, diferentemente de outras regiões, exceto a região Nordeste, em que existe proporcionalidade entre a população e a distribuição de doutores. Outro indicador que comprova esta distorção são as políticas públicas destinadas à pesquisa e a pós-graduação que deveriam minimizar os problemas regionais. Não obstante, com a criação em 2017 do Programa Capes/Print, com foco na internacionalização da pós-graduação, nenhuma instituição da região Norte foi contemplada com recursos (FOPROP, 2020).

De todo modo, as regiões brasileiras evidenciam discrepâncias em distintos indicadores. No que tange à educação é bem significativa, conforme apresentado pelo Atlas de Desenvolvimento Humano (2017), no qual se verifica que todos os estados das regiões Norte e Nordeste estão atrás das regiões Sul, Sudeste e Centro-oeste. Amapá, Roraima, Tocantins e Ceará estão na faixa de desenvolvimento médio $(0,600-0,699)$, enquanto os demais estados estão na faixa de desenvolvimento baixo $(0,500-0,599)$.

\footnotetext{
${ }^{1}$ Por exemplo, no caso da internacionalização universitária, é evidente o quanto é mensurada por indicadores de difusão internacional relacionados às publicações em periódico estrangeiro, preferencialmente com Qualis A e no idioma inglês (SANTIN; VANZ; STUMPF, 2015). Não obstante, apesar deste avanço, os indicadores ainda refletem certa obscuridade e, com isso, emergem discussões acerca de sua capacidade de mensurar a qualidade educacional com parâmetros de internacionalização (LEAL; STALLIVIERI; MORAES, 2017).

${ }^{2}$ Prova disso é que apenas $8 \%$ das universidades do mundo estão inclusas em algum tipo de ranking acadêmico (UNESCO, 2013).
} 
Nesse contexto, emergem as perguntas que orientam este trabalho: Como as universidades podem ser medidas para além dos rankings acadêmicos? Por que as universidades da região Norte não estão presentes nos rankings acadêmicos mais consolidados? Como a aplicação dos Objetivos de Desenvolvimento Sustentável nos rankings acadêmicos pode interferir sobre o que se considera qualidade no ensino superior? De modo a responder essas questões problematizadoras tem-se como objetivo analisar os rankings acadêmicos e suas relações com os ODS em um estudo de caso na Universidade Federal do Tocantins, localizada na região Norte brasileira.

O pressuposto deste estudo é que devido a UFT estar localizada na região Norte do país, em que predomina maiores desigualdades socioeconômicas e tecnológicas que as demais regiões, contribui para sua exclusão nos tradicionais rankings acadêmicos. Por sua vez, acreditase que com o novo ranking, baseado nas premissas dos ODS, pode haver diminuição da assimetria que existe entre as universidades brasileiras.

\section{Metodologia}

Este artigo foi desenvolvido a partir de dados secundários obtidos na internet sobre estudos que tratam acerca dos rankings acadêmicos e suas relações com os ODS no contexto da região Norte do país e da UFT. Para as análises, utilizou-se de artigos científicos, dissertações e teses, além de notícias divulgadas na mídia mapeadas no Google Scholar, no dia 11 de fevereiro de 2021, com as strings: "Ranking and UFT and ODS". Dos 39 mapeados, utilizou-se de 9 que diretamente abordam o objetivo desta proposta, cujos estudos foram adotados para a construção das próximas seções de análise.

Justifica-se a análise de dados secundários por ser uma técnica que possibilita ampliar a compreensão dos objetos empíricos que necessitam de contextualização, de acordo com suas especificidades, pois cada documento tem sua identidade própria e entre si podem proporcionar diálogo (LÜDKE; ANDRÉ, 1986).

\section{Os rankings acadêmicos e suas relações com os Objetivos do Desenvolvimento Sustentável}

Criado com a finalidade de mensurar os impactos na educação superior como, por exemplo, a produção científica, qualidade do ensino e o estreitamento das universidades com o 
setor produtivo; os rankings acadêmicos globais têm influenciado substancialmente o direcionamento, a adequação e a formulação de políticas governamentais para as universidades. Contudo, apesar deste avanço, os indicadores e seus respectivos rankings acadêmicos ainda refletem certa obscuridade e, com isso, emergem questionamentos acerca de sua capacidade de mensurar adequadamente a qualidade educacional a partir de métricas estatísticas (LEAL; STALLIVIERI; MORAES, 2017).

Com outra lógica de se pensar a qualidade educacional, encabeçada pela Organização das Nações Unidades (ONU) foram criados os Objetivos de Desenvolvimento Sustentável com a finalidade de promover a erradicação da pobreza em todas as suas formas e dimensões até 2030, pela qual inclui dentre os 17 ODS propostos o de assegurar a educação inclusiva e equitativa de qualidade, e promover oportunidades de aprendizagem ao longo da vida para todos (ODS 4), tema explorado neste artigo.

Com isso, ao invés de medir o quantitativo de artigos publicados em revistas científicas de alto impacto ${ }^{3}$, em periódico internacional e no idioma inglês ou por meio do mérito acadêmico, passam a serem analisados indicadores como o de discentes de baixa renda e com deficiências, saúde mental dos discentes, igualdade de gênero (ao incentivar que mulheres alcancem cargos de liderança e com adequada remuneração), liberdade acadêmica aos pesquisadores e até mesmo discentes que são os primeiros de suas famílias a cursar o ensino superior (SOUSA, 2019). Righetti (2019) aponta que existem aproximadamente 20 rankings acadêmicos mundiais, no entanto, nenhum considera os aspectos supracitados para avaliar a qualidade das universidades.

Este novo ranking de caráter mais social, foi criado em 2019 pelo Times Higher Education (THE), e é responsável também pelo tradicional e conceituado ranking de universidades mundiais criado em 2004, denominado de Times Higher Education World University Rankings. Nesse novo ranking, denominado de Times Higher Education Impact Rankings, a proposta é avaliar o impacto das universidades no combate à pobreza, o fomento a igualdade de gênero e ações de mitigação climática, dentre outras temáticas atreladas as metas dos ODS. A ideia central é estimular as universidades a estarem alinhadas com os princípios orientadores dos ODS, ao considerar que o maior alinhamento proporcionará maior impacto na sociedade. Trata-se, assim, de avaliar o impacto que as universidades de fato promovem na

\footnotetext{
${ }^{3}$ Por publicações de alto impacto entende-se que são aquelas que estão inseridas em indicadores bibliométricos de referência de determinada área do conhecimento para a avaliação dos Programas de Pós-graduação, como o Qualis-Capes, Journal Citations Report (JCR), H5 do Google Scholar, dentre outros (SOUSA; FUZA, 2020).
} 
sociedade em suas diferentes facetas. Com isso, emerge a discussão sobre o significado das universidades de qualidade: são aquelas com intensa produção técnico-científica e sua internacionalização ou são as que contribuem para um mundo melhor com menos desigualdades? (RIGHETTI 2016; 2019).

Com uma metodologia estruturada, O THE utiliza indicadores calibrados para fornecer comparações abrangentes e equilibradas pautadas em quatro variáveis analíticas: pesquisa, administração, extensão e ensino (Quadro 1). A ideia é avaliar o desempenho da universidade ao relacionar suas ações com os ODS.

Quadro 1 - Características das variáveis analíticas utilizadas na metodologia do THE University Impact.

\begin{tabular}{|l|l|}
\hline Variável analítica & \multicolumn{1}{|c|}{ Explicação } \\
\hline Pesquisa & $\begin{array}{l}\text { As universidades podem entregar os resultados dos ODS ao criar pesquisas } \\
\text { inerentes às metas propostas. }\end{array}$ \\
\hline Administração & $\begin{array}{l}\text { Os funcionários, professores e alunos das universidades são administradores } \\
\text { que têm papel significativo na execução dos ODS. }\end{array}$ \\
\hline Extensão & $\begin{array}{l}\text { Os trabalhos que as universidades desenvolvem com as comunidades locais, } \\
\text { regionais, nacionais e internacionais tornam-se preponderantes para o } \\
\text { impacto pautado na sustentabilidade ao ser transferido o conhecimento } \\
\text { gerado. }\end{array}$ \\
\hline Ensino & $\begin{array}{l}\text { A qualidade do ensino aumenta a perspectiva de que haja profissionais } \\
\text { qualificados e conscientes da importância do cumprimento com os ODS. }\end{array}$ \\
\hline
\end{tabular}

Fonte: Adaptado de THE (2020).

Cada universidade envia os dados que tenham melhor aderência quanto ao cumprimento do alcance das metas propostas pelo ODS. Todas as universidades são pontuadas pelo ODS 17 (parcerias e meios de implantação) e no mínimo mais outros três ODS para serem incluídas na classificação geral deste ranking (THE, 2020).

Os resultados deste novo ranking baseado nas premissas dos ODS apontam que as universidades reconhecidas por sua excelência acadêmica em rankings tradicionais, ou seja, que valorizam primordialmente a abrangência das produções acadêmicas, dentre outros requisitos produtivistas, principalmente as do Reino Unido e Estados Unidos, não se mantém de acordo com o que é levado em conta com as novas variáveis avaliativas. Em outras palavras, as universidades que estão em desvantagem na classificação do ranking tradicional conseguem se destacar nesta nova proposição vinculada aos ODS. Prova disso é a Universidade de Auckland da Nova Zelândia, classificada no agrupamento das posições de 201 a 250 do ranking tradicional 
do THE em 2018, lidera pelo segundo ano consecutivo o Times Higher Education Impact Rankings em 2019, seguida por outras universidades do Canadá, Reino Unido, Suécia, Itália e Hong Kong (RIGHETTI, 2019).

Por sua vez, no Brasil, não é diferente. As universidades também estão mais bem classificadas nesse ranking em relação aos rankings de avaliações tradicionais. Em 2019, foram 30 universidades brasileiras listadas dentre as 200 melhores do mundo ao se comprometerem com o desenvolvimento sustentável. Destaque para a Universidade de São Paulo (USP) que ocupou a 14a posição contribuindo principalmente para o ODS 1 (erradicação da pobreza) através de suas pesquisas relacionadas à esta temática e de intervenções com foco no combate à miséria, além do ODS 15 (vida na terra) com ações direcionadas para a preservação de ecossistemas terrestres. Vale destacar também que a USP foi a universidade brasileira que teve melhor destaque na posição do ranking do THE, figurando entre as 300 melhores do mundo. Assim, a USP se destaca ao ir ao encontro com as métricas dos diferentes rankings acadêmicos, seja aquele mais voltado para a produtividade ou aquele voltado para os aspectos sociais (SUMARES, 2020).

Outro ponto importante a ser revelado é que o Brasil ficou entre os cinco países com maior número de universidades na lista. A liderança ficou com Japão (63), seguido por Rússia (47), Turquia (37), Reino Unido (34), Brasil (30), Estados Unidos (23), Canadá (19), Austrália (21) e China (9) (THE, 2020).

Adiciona-se a essa constatação o fato que dentre as 30 universidades brasileiras listadas, apenas uma é da região Norte. Cita-se a Universidade Federal do Pará (UFPA) que está no agrupamento entre as posições 201 a 300 com maior impacto social (THE, 2020). Isto revela que ainda continuam a existir assimetrias entre as universidades do Norte em relação às demais regiões brasileiras.

\section{Os rankings e sua relação com a Universidade Federal do Tocantins}

A Universidade Federal do Tocantins localiza-se no estado mais novo da federação, o Tocantins, criado em 1988 com a promulgação da Constituição brasileira. Já a UFT foi criada no ano de 2000, a partir da transferência dos cursos e da infraestrutura da Universidade do Tocantins (Unitins). Sua localização estratégica no Norte do país com sua fauna e flora peculiar, 
a credencia como ambiente em potencial para disseminar a ciência e tecnologia no contexto da Amazônia Legal (LAGE, 2015).

A UFT se destaca dentre as universidades da região Norte por ter sido a primeira a estabelecer cotas para indígenas em seus processos seletivos para cursos de graduação e por possuir o curso de Engenharia Ambiental mais antigo do país (UFT, 2021). Apesar de ser uma instituição de ensino recém-criada em comparação com as demais instituições públicas brasileiras mais consolidadas, a UFT já vem se destacando nos rankings acadêmicos.

No anual Ranking Universitário Folha (RUF) que inclui os aspectos pesquisa, ensino, internacionalização, inovação e mercado, a UFT ficou na posição 115a de um total de 197 universidades avaliadas em 2019 (Quadro 1), subindo três posições em relação ao ano anterior. Destaque para os cursos de agronomia (22ㅇ), zootecnia (33으) e engenharia ambiental (36으. Em relação à região Norte, a UFT está atrás apenas da Universidade Federal do Pará (UFPA) que tem mais de 50 anos e a Universidade do Amazonas (UFAM) que é centenária (RUF, 2019).

Quadro 1 - Notas da UFT no RUF 2019

\begin{tabular}{|l|c|c|c|c|c|}
\hline & Pesquisa & Ensino & Internacionalização & Inovação & Mercado \\
\hline Posição & 900 & 150 o & 110 o & $72 \circ$ & 88 o \\
\hline
\end{tabular}

Fonte: RUF (2019).

Em 2020, a UFT foi inserida na lista das melhores universidades da América Latina e do Caribe pela Times Higher Education. Já em relação ao Times Higher Education World University Rankings, a UFT foi avaliada recentemente, em abril de 2021, quanto sua contribuição junto aos ODS. A UFT ficou entre as 1001 melhores instituições de ensino superior do mundo, destaque para os ODS: Cidades e Comunidades Sustentáveis (401-600), Energia Limpa e Acessível (401600), Saúde e Bem-estar (601-800) e Parceria para os Objetivos (601-800) (UFT, 2021).

Isto significa que a instituição está valorizando a implantação das boas práticas para alcance dos ODS. Pode ser citado que a UFT é pioneira na região ao propor políticas institucionais de ações afirmativas de defesa e promoção dos direitos humanos e da igualdade étnico racial. Citam-se os quilombolas e indígenas que são beneficiários de cotas para ingresso em cursos de graduação e pós-graduação e podem usufruir, como os demais estudantes em situação de vulnerabilidade, do Programa Bolsa Permanente, no valor de R\$400,00/mês. Tratase, assim, de dar maiores condições a dois públicos endógenos do Tocantins e que de alguma forma são excluídos da sociedade. 
Portanto, os dados secundários encontrados indicam que a UFT está se inserindo gradativamente nos rankings acadêmicos, de acordo com as métricas tradicionais de avaliação, e concomitantemente está desenvolvendo ações direcionadas também ao favorecimento de impactos sociais positivos que podem ser proporcionados a partir de sua contribuição no ensino, pesquisa e extensão alinhado as metas dos ODS.

\section{Considerações finais}

Este estudo concluiu que existe, nos últimos anos, maior interesse da sociedade por informações sobre as universidades. Isto proporcionou a proliferação de rankings acadêmicos como forma de analisar a qualidade do ensino, cada qual com sua metodologia. Ao se afastar das métricas que mensuram, em suma, a produtividade no meio universitário, foi criado o Times Higher Education Impact Rankings que analisa o desempenho global das universidades em relação aos ODS, como alternativa de avaliação das universidades menos tradicionais, que recebem menor quantidade de recursos de agências financiadoras e/ou aquelas que estão localizadas em regiões menos desenvolvidas e com aguçada desigualdade.

A conclusão que se chega é que mesmo diante da criação de um ranking atrelado aos ODS, as universidades da região Norte permanecem excluídas. Isto ocorre por não conseguirem, ainda, direcionar todos os esforços no intuito de potencializar as ações que promovam o que é considerado desenvolvimento pelo novo ranking, principalmente por estarem localizadas em uma região em que vigoram maiores desigualdades socioeconômicas e tecnológicas.

Por sua vez, a UFT está em processo de inserção em alguns rankings acadêmicos e, também, no Times Higher Education Impact Rankings. A sua recente inserção no ranking dos ODS poderá obter resultados mais promissores neste do que nas demais classificações acadêmicas. Fato este justificado pela recorrência de ações que promovem a inclusão de indígenas e quilombolas em suas ações afirmativas educacionais, por exemplo. Isto retrata uma forma de reduzir a assimetria que existe entre as universidades brasileiras e o fomento a erradicação da pobreza, que é o princípio orientador dos Objetivos do Desenvolvimento Sustentável. Trata-se, assim, de promover ações para melhorias na condição de vida da população, principalmente do estado do Tocantins e dos estados vizinhos, impactando consideravelmente no Índice de Desenvolvimento Humano (IDH). 


\section{Referências}

ALTBACH, P. The dilemmas of ranking. International Higher Education, n. 42, p. 23, 2015. ATLAS DO DESENVOLVIMENTO HUMANO. Ranking - IDH Educação. 2017. Disponível em: < http://www.atlasbrasil.org.br/ranking>. Acesso em 12 mar. 2021.

OULTON, G; LUCAS, C. What are universities for? Chinese Science Bulletin. v. 26, n.23, p.25062517, 2011.

BRASIL. Lei $n$ ㅇ 9.394, de 20 de dezembro de 1996. Estabelece as diretrizes e bases da educação nacional. Diário Oficial da União, Brasília, DF, 23 dez. 1996.

BRASIL. Lei no 10.861, de 14 de abril de 2004. Institui o Sistema Nacional de Avaliação da Educação Superior - SINAES e dá outras providências. Diário Oficial da União, Brasília, DF, 15 abr. 2004.

FOPROP - Fórum Nacional de Pró-Reitores de Pesquisa e Pós-graduação das IES brasileiras Regional Norte-Amazônia Legal. Carta da Amazônia 2020/2030. 2020. Disponível em: <http://www.foprop.org.br/uploads/downloads/2020 10 20/CARTA-2020-2030-FOPROPNORTE.pdf>. Acesso em: 12 mar. 2021.

LEAL, F. G; STALLIVIERI, L; MORAES, M. C. B. Indicadores de internacionalização: o que os rankings acadêmicos medem? Revista Interacional de Educação Superior, v.4 [54], n.1 p.52-73, 2018.

GONÇALVES, A.; CALDERON, A. I. Academic rankings in higher education: trends of international scientific literature. Revista Diálogo Educacional, v. 17, p. 1125-1145, 2017.

INEP - Instituto Nacional de Estudos e Pesquisas Educacionais Anísio Teixeira. Ministério da Educação. Notas estatísticas do Ensino Superior 2019. INEP: Brasília, 2019. 32p. Disponível em: https://bit.ly/3qXhQoH. Acesso em: 26 fev. 2021.

KING, R. Governing universities globally: organizations, regulation and rankings. Cheltenham, UK; Northampton, MA: Edward Elgar, 2009.

KNIGHT, J. International universities: misunderstandings and emerging models? Journal of Studies in International Education, v. 19, n. 2, p. 1-15. 2015.

LAGE, T. S. R. Políticas de internacionalização da educação superior na região norte do Brasil: uma análise do Programa Ciência sem Fronteiras. 183 f. Dissertação (Mestrado em Desenvolvimento Regional), Universidade Federal do Tocantins (UFT), Palmas, Tocantins, 2015. LÜDKE, M; ANDRÉ, M. E. D. A. Pesquisa em educação: abordagens qualitativas. São Paulo: EPU, 1986.

NUNES, E; FERNANDES, I. Rankings internacionais: a irresistível polêmica em torno de seus sentidos e metodologias. Revista Ensino Superior, n.12, 2014.

OECD - Organização para a Cooperação e Desenvolvimento Econômico. What are the returns on higher education for individuals and countries? 2012. Disponível em:< https://www.oecdilibrary.org/education/what-are-the-returns-on-higher-education-for-individuals-andcountries 5k961l69d8tg-en>. Acesso em 19 mar. 2021. 
PÉREZ-ESPARRELLS, C; LOPEZ, A, g. Rankings de instituciones de educación superior: panorama internacional. Calidad en la Educación, v. 30, p. 328-343, 2009.

UFT - Universidade Federal do Tocantins. Histórico da UFT. 2021. Disponível em: <https://ww2.uft.edu.br/index.php/acessoainformacao/institucional/historia $>$. Acesso em: 13 de mar. 2021.

RIGHETTI, S. Qual é a melhor? Origem, indicadores, limitações e impactos dos rankings universitários. 230f. Tese (doutorado em Política Científica e Tecnológica). Universidade de Campinas (Unicamp), Campinas, 2016.

. ODS aplicados à avaliação educacional reacendem debate: afinal, o que é uma boa universidade? COMCIÊNCIA (Unicamp), v. 208, p. 1, 2019.

RUF - Ranking Universitário Folha. Ranking de Universidades. 2019. Disponível em: <https://ruf.folha.uol.com.br/2019/ranking-de-universidades/principal/>. Acesso em: 19 mar. 2021.

SANTIN, D. M; VANZ, S. A. S; STUMPF, I. R. C. Internacionalização da produção científica em Ciências Biológicas da UFRGS: 2000-2011. TransInformação, Campinas, v.27, n.3, p. 209-218, 2015.

SANTOS, S. M; NORONHA, D. P. O desempenho das universidades brasileiras em rankings internacionais. Em Questão, Porto Alegre, v. 22, n. 2, p. 186-219, 2016.

SOUSA, M. S. C. Práticas de letramentos e o processo de internacionalização de Programas de Pós-graduação na UFT. 129 f. Dissertação (Mestrado em Letras). Universidade Federal do Tocantins, Porto Nacional - TO, 2019.

SOUSA, M. S. C; FUZA, A. F. A temática "internacionalização" e sua relação com o contexto acadêmico. Humanidades \& Inovação, v.7, n. 8, p. 1-17, 2020.

SUMARES, G. Ranking de impacto social coloca USP entre as $\mathbf{2 0}$ melhores universidades do mundo. 2020. Disponível em: < https://www.estudarfora.org.br/ranking-the-metas-onu/>. Acesso em: 19 mar. 2021.

THE - Times Higher Education. THE Impact Rankings 2020: methodology. 2020. Disponível em: <https://www.timeshighereducation.com/impact-rankings-2020-methodology>. Acesso em: 13 mar. 2021.

UNESCO - Organização das Nações Unidas para a Educação, a Ciência e a Cultura. Rankings and accountability in higher education uses and misuses. UNESCO, 2013.

UFT - Universidade Federal Do Tocantins. UFT é listada em ranking internacional que avalia impacto das universidades com os ODS. 2021. Disponível em:

<https://ww2.uft.edu.br/index.php/ultimas-noticias/29411-uft-e-listada-em-rankinginternacional-que-avalia-impacto-das-universidades>. Acesso em: 10 maio 2021.

INCKE, P. University rankings. In: DEHON, C; JACOBS, D; VERMANDELE, C. (orgs). Ranking universities. Editions de L'Universite de Bruxelles, 2009. p.11-26.

VIEIRA, R. C; LIMA, M. C. A busca pelo selo "universidade classe do mundo" e os rankings acadêmicos globais. Interfaces Brasil/Canadá, v.16, n.1, 97-116, 2016. 